\title{
Neuronal Injury Marker ATF-3 Is Induced in Primary Afferent Neurons of Monoarthritic Rats
}

\author{
Diana Nascimento Daniel Humberto Pozza José Manuel Castro-Lopes \\ Fani Lourença Neto \\ Departamento de Biologia Experimental, Faculdade de Medicina do Porto e Instituto de Biologia Molecular e \\ Celular (IBMC), Universidade do Porto, Porto, Portugal
}

\section{Key Words}

Activating transcription factor $3 \cdot$ Calcitonin gene-related peptide - Isolectin B4 - pAkt - Dorsal root ganglia ·

Joint inflammatory pain $\cdot$ Neuronal damage $\cdot$

Immunohistochemistry

\begin{abstract}
Activating transcription factor 3 (ATF-3) expression has been associated with several signaling pathways implicated in cellular stress response in many cell types and is usually regarded as a neuronal damage marker in dorsal root ganglia (DRG). We investigated ATF-3 expression in primary afferents in the monoarthritic (MA) model of chronic inflammatory joint pain. Immunohistochemistry revealed that ATF-3 is highly induced mainly in small and medium neurons, especially at 2 and 4 days of MA in $L_{5}$ DRGs. Colocalization with calcitonin gene-related peptide (CGRP) and isolectin B4 (IB4) demonstrated that ATF-3-immunoreactive cells are mainly peptidergic. The lack of significant differences in ATF-3 and pAkt colocalization indicated that ATF-3 is probably not involved in a pAkt-mediated survival pathway. Anti-inflammatory (ketoprofen) administration failed to reverse ATF-3 induction in MA rats, but significantly increased CGRP expression. These data suggest that ATF-3 expression is definitely involved in MA, actually marking injured neurons. Some degree of neuronal damage seems to occur right
\end{abstract}

from the first days of disease, mainly affecting small-to-medium peptidergic neurons. The intra-articular injection of complete Freund's adjuvant and the generation of a neuroinflammatory environment seem to be the plausible explanation for the local nerve damage.

Copyright $\odot 2011$ S. Karger AG, Basel

\section{Introduction}

Joint inflammation is a major clinical problem and a main cause of debilitating chronic pain, characterized by pronounced mechanical hyperalgesia and persistent pain at rest [1]. Pain mechanisms require the sensitization of primary sensory neurons, whose cell bodies are located in dorsal root ganglia (DRG), and involve several mediators that trigger particular signal-transduction pathways. A great part of these pathways imply the activation of transcription factors, in which gene expression becomes altered. In particular pathological conditions, DRGs are actually responsible for the synthesis of signaling molecules involved in reaction cascades that can ultimately lead to phenomena like survival or regeneration. Elucidation of these related molecular mechanisms is crucial to understand pain transmission processing in an inflammatory condition, bringing into light possibilities for new treatment approaches [2].

\section{KARGER}

Fax +4161306 1234

E-Mail karger@karger.ch

www.karger.com
(C) 2011 S. Karger AG, Basel

1424-862X/11/0194-0210\$38.00/0

Accessible online at:

www.karger.com/nsg
Fani Lourença Moreira Neto

Departamento de Biologia Experimental

Faculdade de Medicina da Universidade do Porto

Alameda Prof. Hernâni Monteiro, PT-4200-319 Porto (Portugal)

Tel. +351 22551 3654, E-Mail fanineto@ med.up.pt 
Activating transcription factor 3 (ATF-3) has been suggested as acting as an 'adaptive response' due to its ability to respond differently accordingly to the cellular context [3]. Its inclusion in anti- and pro-apoptosis mechanisms [4], cell survival [5], regeneration [6] and neuroprotection [7] signaling events has been reported. In a pAkt-mediated survival pathway the ATF-3/c-Jun heterodimer has been suggested to promote nerve elongation and inhibit apoptosis in neurons under death stress such as nerve injury [8]. In pain models, a marked increase in pAkt levels was observed following peripheral nerve injury, whereas carrageenan-induced inflammation induced only slight increases in the number of pAkt-positive DRG neurons without affecting the activated (phosphorylated) protein levels. Despite the significance of these results, a very limited effect of the intradermal carrageenan injection, and the associated inflammatory mechanisms, was demonstrated [9]. However, the role of these biomolecules in pain pathophysiology is still to be unmasked.

Nowadays, ATF-3 is mostly assumed as a neuronal injury marker, after its expression was found to be highly induced in several models of neuropathic pain [1012]. Interestingly, in the collagen-induced arthritis and monoiodoacetate-induced osteoarthritis joint pain models, ATF-3 was also expressed in DRG neurons [13, 14], suggesting some degree of neuronal damage is occurring. However, data on ATF-3 expression in primary afferents in different models of inflammatory pain have not always been consistent [15-17]. For example, intraplantar injection of complete Freund's adjuvant (CFA) did not induce any ATF-3 expression, suggesting there is no neuronal damage associated $[15,16]$.

Taking into account these controversial data and the sparse information available on the role of ATF-3 in pain processing, we aimed to investigate its expression pattern in primary afferents during different timepoints of monoarthritis (MA), a well established model of chronic joint inflammatory pain induced by injection of CFA into the tibiotarsal joint [18]. In order to characterize the neuronal populations most implicated, we further analyzed the ATF-3-expressing cells' size distribution and colocalization with isolectin B4 (IB4) or calcitonin gene-related peptide (CGRP) [19]. CGRP has early been described to potentiate pain signaling from primary sensory neurons to the spinal cord, functioning as a mediator of neurogenic inflammation at the periphery [20]. It has been recognized as a nerve regeneration-promoting peptide, after neuronal damage [21]. More recently, its expression was shown to be either increased or decreased depending on the sciatic nerve injury, indicating that the nature of the peripheral injury has an impact on CGRP expression [22] even affecting different neuronal populations [23]. Thus, possible changes in CGRP expression during MA were also evaluated. In order to investigate the trigger of a possible survival pathway associated to ATF-3 induction in MA, pAkt expression was also analyzed. Additionally, we administered an anti-inflammatory non-selective cyclooxygenase (COX) inhibitor (ketoprofen) in order to evaluate its effect on ATF-3 and CGRP expression during establishment of MA. Parts of this work have been published in abstract form [24, 25].

\section{Materials and Methods}

\section{Animal Handling and MA Induction}

Experiments were carried out in adult male Wistar rats (Charles River Laboratories, France) weighing between 200 and $300 \mathrm{~g}$. A total of 42 animals were used in this study but 2 of them were excluded since they developed signs of polyarthritis (see Results). Animals were housed 2-3 animals per cage under controlled conditions of lighting (12-hour light/12-hour dark cycle) and temperature as well as water and food ad libitum. Efforts were made in order to minimize pain and distress and reduce the number of animals used. All procedures were carried out according to the European Communities Council Directive 86/609/EEC amended by the Directive 2003/65/CE of July 22, 2003 and to the ethical guidelines for investigation of experimental pain in animals [26]. MA was induced by injecting $50 \mu \mathrm{l}$ of CFA into the left tibiotarsal joint [18] under isoflurane anesthesia (5\% for induction, $2.5 \%$ for maintenance). CFA was prepared as previously described [27] and MA animals were allowed to survive for 2, 4, 7 and 14 days. Control animals were injected with $50 \mu$ lof CFA vehicle (composed by the same reagents as CFA except for Mycobacterium butyricum) and allowed to survive for 2 days. To minimize fear-motivated behaviors, animals were habituated to the experimenter for several days before CFA injection and during the progression of MA. The evolution of the inflammatory reaction was monitored daily and was scored according to Castro-Lopes et al. [28]. This score takes in consideration the inflammatory signs of the injected ankle and reduction of the locomotor activity. Score 0 means no inflammatory signs. Score 1 indicates the presence of minor changes such as redness and swelling and score 2 denotes more intense swelling and some avoidance of passive movements. In score 3, additionally, rats show reluctance to place weight over the affected limb. In score 4 , there is severe inflammation with persistent flexion of the affected limb and repercussion over the motor activity of the animal. In order to better evaluate the severity of inflammation, the diameter of the animals' affected paw was also measured, right before sacrificing them.

\section{Immunohistochemistry}

All animals were perfused through the ascending aorta with $250 \mathrm{ml}$ of oxygenated Tyrode's solution followed by $750 \mathrm{ml}$ of paraformaldehyde $4 \%$ in phosphate buffer $(\mathrm{PB}) 0.1 \mathrm{M}$, after intraperitoneal anesthesia with chloral hydrate $35 \%(0.1 \mathrm{ml} / 100 \mathrm{~g}$ of animal weight). The ipsi- and contralateral DRGs corresponding to spinal segments $\mathrm{L}_{3}, \mathrm{~L}_{4}$ and $\mathrm{L}_{5}$ were removed and post-fixed in the same 
fixative solution for $4 \mathrm{~h}$ and then cryoprotected overnight (sucrose $30 \%$ in phosphate buffer saline (PBS) $0.1 \mathrm{M}$ ). The DRGs, either belonging to CFA vehicle-injected controls or inflamed CFA-injected animals with 2, 4, 7 and 14 days of MA, were cut sequentially into $14-\mu \mathrm{m}$ sections in a freezing cryostat $\left(-20^{\circ} \mathrm{C}\right)$. The material was collected into poly-L-lysine-coated slides, air dried and stored at $-20^{\circ} \mathrm{C}$ until immunohistochemistry was performed.

After the blocking step in the correct normal serum (normal goat serum for ATF-3 single and ATF-3/pAkt double immunohistochemistry and normal horse serum for triple immunoreaction against ATF-3, CGRP and IB4), in order to avoid unspecific bindings, slides containing every fifth section of each DRG were incubated for $48 \mathrm{~h}$ at $4{ }^{\circ} \mathrm{C}$ in appropriate primary antibodies. Sections were then incubated for $1 \mathrm{~h}$ at room temperature in the suitable secondary antibodies. For the single immunolabeling against ATF-3, $\mathrm{L}_{3}, \mathrm{~L}_{4}$ and $\mathrm{L}_{5}$ ipsi- and contralateral DRG sections ( $\mathrm{n}=5$ rats for each experimental group) were incubated in polyclonal rabbit anti-ATF-3 (1:500; C-19: sc-188; Santa Cruz Biotechnology, Inc.). Detection was achieved by Alexa 594 goat-anti-rabbit (1:1,000; A11012; Molecular Probes, USA). For the double immunolabeling against ATF-3 and pAkt, $\mathrm{L}_{5}$ ipsilateral DRG sections ( $\mathrm{n}=4$ rats for controls; $\mathrm{n}=5$ rats for each of the other experimental groups) were incubated in polyclonal rabbit anti-ATF-3 (1:500) and monoclonal mouse anti-pAkt $(1: 1,000 ; 5106 S$; Cell Signalling). Detection was achieved by Alexa 594 goat-anti-rabbit $(1: 1,000)$ for ATF-3, and Alexa 488 goat-anti-mouse (1:1,000; A11029; Molecular Probes) for pAkt. For the triple immunolabeling against ATF-3, CGRP (neuronal marker for peptidergic primary afferents) and IB4 (neuronal marker for non-peptidergic primary afferents), $L_{5}$ ipsilateral DRG sections ( $n=5$ rats for controls and the $4 \mathrm{~d} M A ; \mathrm{n}=4$ rats for each of the other experimental groups) were firstly incubated in polyclonal rabbit anti-ATF-3 (1:500) and polyclonal sheep anti-CGRP (1:4,000; ab22560; Abcam, Cambridge, UK). Detection was achieved using Alexa 488 donkey anti-rabbit (1:1,000; A21206; Molecular Probes) for ATF-3, and Alexa 568 donkey anti-sheep (1:1,000; A21099; Molecular Probes) for CGRP. Slides with the DRG sections were afterwards incubated in the biotin-conjugated IB4 from Bandeiraea simplicifolia (1:1,000; L2140; Sigma-Aldrich) specially diluted in a PBS with Triton X-100 (PBST) solution (without normal serum), containing magnesium chloride $\left(\mathrm{MgCl}_{2}\right)$, manganese chloride $\left(\mathrm{MnCl}_{2}\right)$ and calcium chloride $\left(\mathrm{CaCl}_{2}\right)$ in a 1:50 final concentration, for one night, at room temperature. Detection was achieved by incubation in Streptavidin 350 (1:200; Jackson ImmunoResearch Laboratories, Inc.). As specificity controls for each immunoreaction, slides were processed in a similar way as described above but without incubation in primary antibody.

At the time of analysis, glass slides containing the immunoreacted sections were coverslipped with glycerol prepared with PBS $0.4 \mathrm{M}$ and visualized under fluorescence microscope.

\section{Ketoprofen Treatment}

MA was induced in rats as described above, and ketoprofen, a non-steroidal anti-inflammatory COX inhibitor drug, was daily administered subcutaneously $(5 \mathrm{mg} / \mathrm{kg}$ of rat $/ 24 \mathrm{~h}$ ). In a first experimental group, ketoprofen daily treatment started at day 0 , when MA was induced by CFA injection, to diminish the development of the neuroinflammatory environment right from the beginning. In a second experimental group, ketoprofen daily treatment started only after day 2 of MA, as we knew that ATF-3 was already significantly induced in $\mathrm{L}_{5}$ ipsilateral DRGs at this time- point. A control group of rats was injected with CFA into the tibiotarsal joint to induce MA, and received a daily subcutaneous saline injection. All animals were perfused at 4 days of MA $(n=5$ rats for each experimental group), the peak of ATF-3 expression, and the $\mathrm{L}_{5}$ ipsi- and contralateral DRGs were dissected and further processed for immunohistochemistry against ATF-3 ( $\mathrm{n}=4$ rats for each experimental group) and CGRP $(n=5$ rats for each experimental group) as described above. All animals were evaluated for the severity of inflammatory symptoms by using an appropriate score [28] and by measuring the diameter of the inflamed paw as described above.

\section{Data Analysis}

\section{Cell Counting}

Immunohistochemistry analysis was performed in a blinded manner using a fluorescence microscope (AxioImager Z1; Zeiss) coupled to a digital camera (AxioCam MRm) and computer image software (AxioVision 4.6) to grab the images. For the photomicrographs, the acquisition conditions such as amplification of the objective, light intensity, contrast and hue were maintained constant.

For ATF-3 single labeling, all immunoreactive (IR) cells were counted in every fifth section of the ganglion and divided by the total of tissue sections containing neuronal cell bodies present in the respective glass slides (approximately 9-12 sections per DRG). ATF-3 nuclear labeling is very clear and undoubtedly easy to detect, so only cells where nuclei were visualized were considered. For quantification of pAkt-IR cells in ATF-3+pAkt double labeling, a threshold was established based on labeling for fibers, and only cells with a signal above that threshold were considered as positive. This was performed with the help of the Image ${ }^{\circledR}$ version 1.37 (free access software) computer program. At least 350 total cells with visible nuclei per rat (corresponding more or less to a sample of 1,500 cells per experimental group) were randomly chosen from 8 to 9 (every fifth) sections of the same DRG and were counted, as similarly described before $[23,29]$. pAkt immunoreactivity was expressed as the percentage of pAkt-IR cells in the total number of counted neurons. Colocalization between pAkt and ATF-3 was expressed as the percentage of double IR cells in the total number of ATF-3-IR cells (ATF-3 neuronal population). For the triple immunolabeling against ATF-3, CGRP and IB4, quantification was done also randomly in $8-9$ sections by using an approach similar to that described for pAkt. Depending on the marker this corresponded to 400-750 labeled neurons per experimental group. Thus, in order to evaluate the expression of ATF-3 and the two markers individually, IR cells (for each of the three molecules) were counted and divided by the total number of selected neurons. To obtain percentages of colocalization between ATF-3 and CGRP or ATF-3 and IB4, double labeled cells were also counted and divided by the total number of ATF-3-expressing neurons [15]. This same random selection of at least 350 total cells in 8-9 sections of the same DRG and a similar calculation of percentage values was used in single CGRP labeling for both untreated MA and ketoprofen-treated MA animals.

\section{Cell Size Distribution}

Areas of all the ATF-3-IR cells were measured in all experimental groups $(n=4$ rats for $7 d M A ; n=5$ rats for each of the other experimental groups), in order to evaluate possible switches in ATF-3-positive neuronal population(s) along MA progression. CGRP-positive neuron areas were measured in the same IR cells 
randomly selected for cell counting ( $\mathrm{n}=5$ rats for controls and the $4 \mathrm{~d}$ MA; $\mathrm{n}=4$ rats for each of the other experimental groups). IR cells with visible nuclei were manually outlined using a computer mouse and the cross-sectional area was determined using the ImageJ version 1.37 (free access software) [29, 30]. Cell areas were grouped into three categories: small $\left(<600 \mu \mathrm{m}^{2}\right)$, medium (600$\left.1,200 \mu \mathrm{m}^{2}\right)$ and large neurons $\left(>1,200 \mu \mathrm{m}^{2}\right)$ as described by Noguchi's group [31].

\section{Statistical Analysis}

Statistical analysis was performed by using GraphPad Prism $5^{\circledR}$ (GraphPad Software) and SPSS 13.0. One-way analysis of variance (one-way ANOVA) was performed for all data to investigate significant differences between experimental groups, followed by the appropriate post-hoc tests when the level of significance was considered as $\mathrm{p}<0.05$.

Bonferroni post-hoc test was used to discriminate differences between all experimental groups in ATF-3 and pAkt single immunolabeling, ATF-3 and pAkt colocalization and also in triple immunoreactions against ATF-3, CGRP and IB4. This post-hoc test was also used to evaluate differences in paw diameter. The Newman-Keuls multiple comparison test was used to discriminate differences in cell size distribution of ATF-3- or CGRP-positive cells. In order to find differences in ATF-3 or CGRP expression between the non-treated $4 \mathrm{~d}$ MA rats (non-ketoprofen group), the $4 \mathrm{~d}$ MA rats treated during 2 days (2d ketoprofen) and the $4 \mathrm{~d}$ MA rats receiving ketoprofen from day 0 (4d ketoprofen), the Newman-Keuls multiple comparison post-hoc test was also used.

\section{Results}

\section{MA Was Successfully Induced}

All CFA-injected rats showed severe inflammatory symptoms such as swelling, redness and avoidance to put weight over the inflamed paw. This was reflected in mean inflammatory scores near 4 (maximum score), right after the second day of MA (3.67 \pm 0.12$)$, maintaining this condition up to the 14th day (3.75 \pm 0.13$)$ in accordance with our previous work $[32,33]$. Controls showed insignificant mean scores $(0.67 \pm 0.11)$, probably due to local trauma caused by the injection procedure. MA animals' inflamed paws showed also significantly $(\mathrm{p}<0.001)$ greater diameters at all inflammatory timepoints (1.24 \pm $0.03 \mathrm{~cm}$ for $4 \mathrm{~d} \mathrm{MA}$, for example) than controls $(0.59 \pm$ $0.02 \mathrm{~cm})$. Therefore, MA was successfully and homogenously induced in all animals injected with CFA, as they were all showing similar physiological responses at each timepoint of disease.

Some animals in the latter phases of the condition (14 days of MA majorly) may develop polyarthritis, with the contralateral non-injected paw and sometimes the tail starting to show inflammatory signs, as described by Butler et al. [18]. In our study, all animals showing signs of polyarthritis ( $\mathrm{n}=2$ rats) were immediately excluded.

\section{ATF-3 Is Highly Induced in Primary Afferents during}

$M A$

ATF-3 expression was induced in the ipsilateral DRG neurons of MA rats at all timepoints of inflammation (fig. 1b, c). The number of ATF-3-IR cells per tissue slice was significantly increased at 2 and 4 days of MA and it started diminishing after that period, although at 7 and 14 days of inflammation ATF-3 was still induced. Increases were observed in all DRG levels studied $\left(\mathrm{L}_{3}, \mathrm{~L}_{4}\right.$ and $\mathrm{L}_{5}$ ), but were more considerable in $\mathrm{L}_{4}$ and $\mathrm{L}_{5}$ ganglia, while DRGs from control rats showed no significant ATF-3 expression (fig. 1c). Statistical significant differences were reached at 4 days of MA for the $\mathrm{L}_{4}$, and at 2 and 4 days of MA for the $\mathrm{L}_{5}$ DRGs $(\mathrm{p}<0.05$; one-way ANOVA followed by Bonferroni post-hoc test; fig. 1c). No significant expression of ATF-3 was observed in either controls or contralateral DRGs in any experimental group (fig. 1a).

\section{ATF-3-Expressing Cells Are Mainly Small-to-Medium Neurons}

Measurement of the cells' areas indicated that ATF-3 is majorly expressed by small- to medium-sized neurons during MA. This is especially obvious for the later stages of disease, since the number of ATF-3-expressing cells belonging to the large-sized group of neurons $(>1,200$ $\left.\mu \mathrm{m}^{2}\right)$ is much lower than that of small $\left(<600 \mu \mathrm{m}^{2}\right)$ and medium cells $\left(600-1,200 \mu \mathrm{m}^{2}\right)$ in the 7 and $14 \mathrm{~d}$ MA rats (fig. 1d). Statistical analysis of significant differences in the number of ATF-3-IR cells between each of the three distinct sizes within the same experimental group revealed that they were only significant for the $14 \mathrm{~d}$ MA animals $(1.3 \pm 0.2,2.2 \pm 0.4$ and $0.3 \pm 0.1$ ATF-3-IR cells/ tissue slice for sizes $<600,600-1,200$ and $>1,200 \mu \mathrm{m}^{2}$, respectively). Greater statistical significance was reached for differences between the number of medium- and large-sized ATF-3-IR cells ( $\mathrm{p}<0.001$, one-way ANOVA followed by Newman-Keuls multiple comparison test), reflecting the lower number of ATF-3-expressing cells with a cross-sectional area $>1,200 \mu \mathrm{m}^{2}$ (fig. 1d).

\section{Expression of ATF-3 Is Higher in Peptidergic than in} Non-Peptidergic Neurons

Analysis of ATF-3 single labeled cells (fig. 2a) corroborated data previously obtained confirming that MA induces ATF-3 expression in ipsilateral primary afferent neurons (table 1). Thus, the ATF-3 expression was always higher in MA animals when compared to controls which showed no ATF-3 expression. As in the previous data, the $2 \mathrm{~d}$ (with $6.1 \pm 1.0 \%$ ATF-3-IR cells) and $4 \mathrm{~d}(8.0 \pm$ 


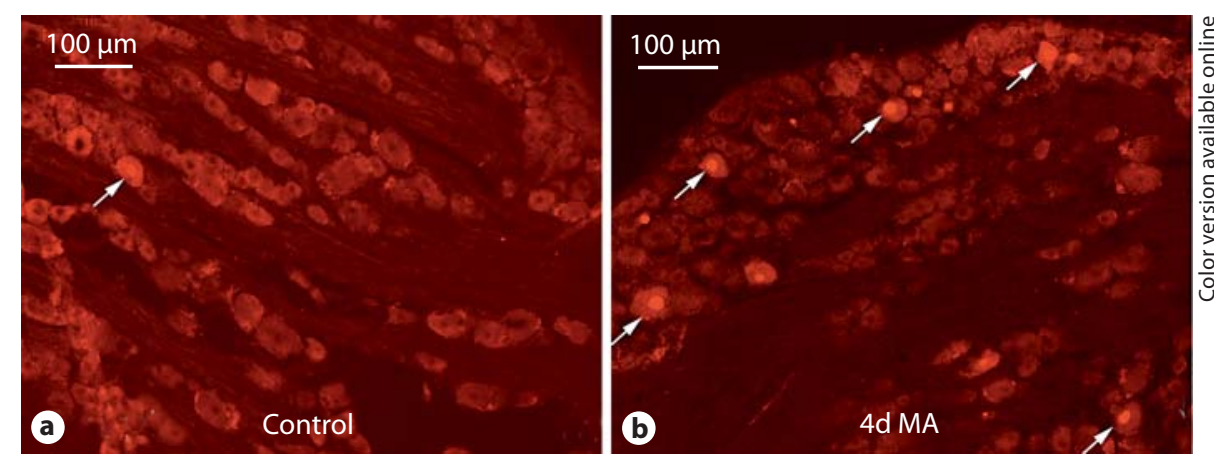

Fig. 1. ATF-3 expression in primary afferents of MA rats. a, b Fluorescent microscope photo images depicting single immunolabeling for ATF-3 in $\mathrm{L}_{5}$ ipsilateral DRG neurons from a non-inflamed control rat (a) and from a CFA-injected rat with 4 days of MA (b). ATF-3-expressing cells are shown with the red-labeled nuclei (white arrows). Scale bar represents 100 $\mu \mathrm{m}$. c The number of ATF-3-IR cells/tissue slice was significantly increased (in comparison to controls) at $4 \mathrm{~d} \mathrm{MA}$ for the $\mathrm{L}_{4}$ ganglia and at $2 \mathrm{~d}$ and $4 \mathrm{~d} \mathrm{MA}$ for the $\mathrm{L}_{5}$ ganglia. Although statistical significances were not reached for other MA timepoints, inflamed animals showed always higher expression than controls. Values shown as mean \pm SEM. * represents $\mathrm{p}<0.05$ (oneway ANOVA followed by Bonferroni posthoc test). $n=5$ rats per experimental group. d Cell size distribution of ATF-3-expressing cells, as described in Fukuoka et al. [31], revealed they were mainly small $\left(<600 \mu \mathrm{m}^{2}\right)$ and medium-sized (between 600 and $1,200 \mu \mathrm{m}^{2}$ ) neurons at all timepoints of MA. This is especially evident for the later stages of disease (14d MA) where significant differences were found for different size categories. ${ }^{*}$ represents $\mathrm{p}<0.05$ and ${ }^{* *}$ represents $\mathrm{p}<0.001$. One-way ANOVA followed by Newman-Keuls multiple comparison test for evaluating differences in the numbers of IR cells between each size within each experimental group. $\mathrm{n}=5$ rats for all groups except for $7 \mathrm{~d} \mathrm{MA}$ with $\mathrm{n}=4$ rats.
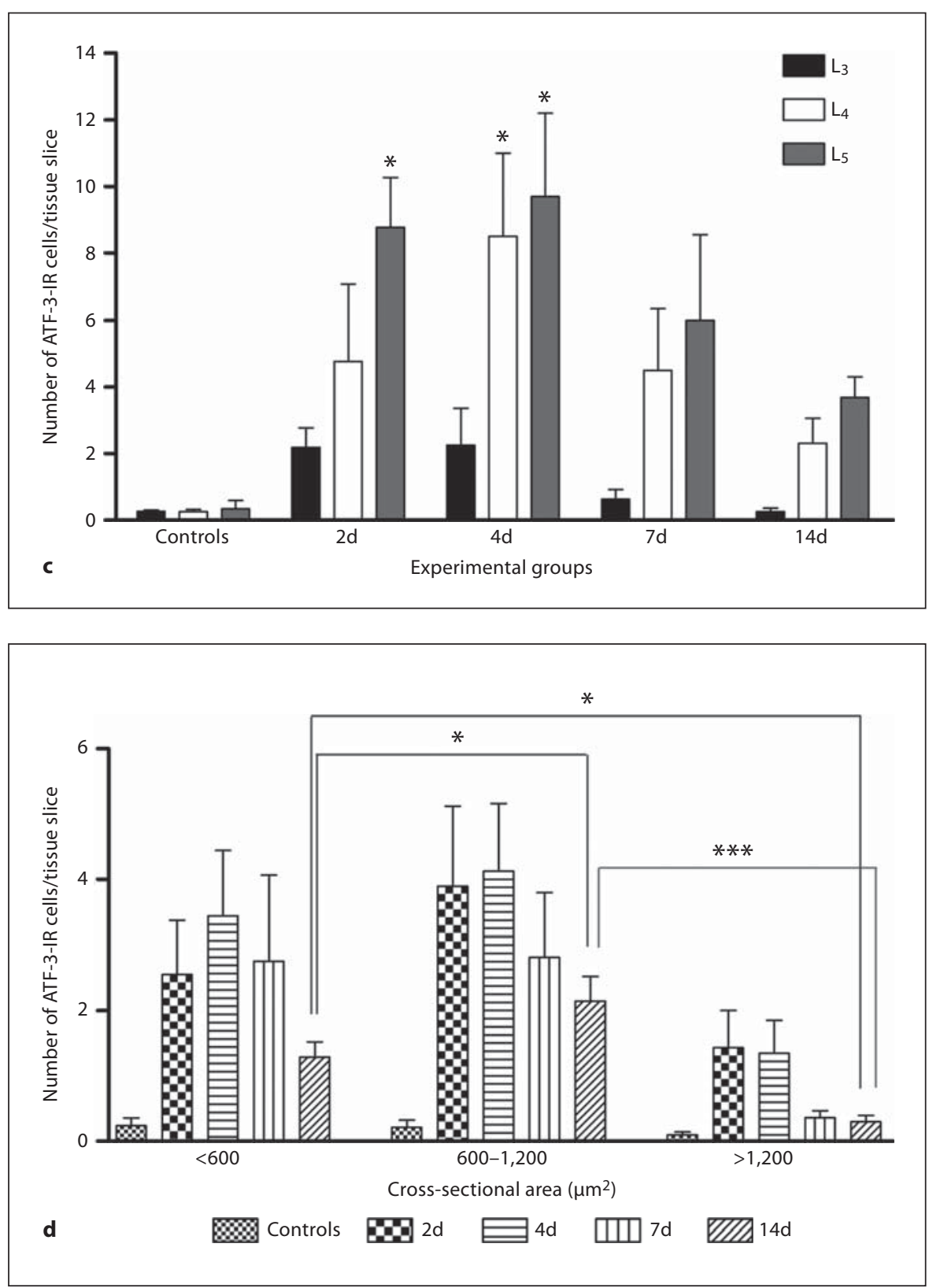
Fig. 2. ATF-3 colocalization with CGRP and IB4, and CGRP expression pattern. a-d Fluorescent microscope photo images depicting single immunolabeling for ATF3 (green nucleus) (a), CGRP (red cytoplasms) (b), IB4 (blue cytoplasms) (c) and triple immunolabeled cells (d), in a $\mathrm{L}_{5}$ ganglia of a $4 \mathrm{~d}$ MA animal (20 $\times$ magnification). Scale bars represent $50 \mu \mathrm{m}$. e Cell size distribution of CGRP-expressing cells in the whole DRG neuronal population revealed no neuronal population switch during MA, also reinforcing that these peptidergic neurons are mainly small-sized. Values shown in percentages as mean \pm SEM. * were used to point significant differences between small-sized neurons and the other two size categories (medium and large); " were used to point significant differences between medium- and large-sized neurons. \# represents $\mathrm{p}<0.05$ and ***/\#\#\# represents $\mathrm{p}<0.001$. One-way ANOVA followed by Newman-Keuls multiple comparison test. $\mathrm{n}=5$ rats for controls and $4 \mathrm{~d} \mathrm{MA}$, and $\mathrm{n}=4$ for each of the other experimental groups.
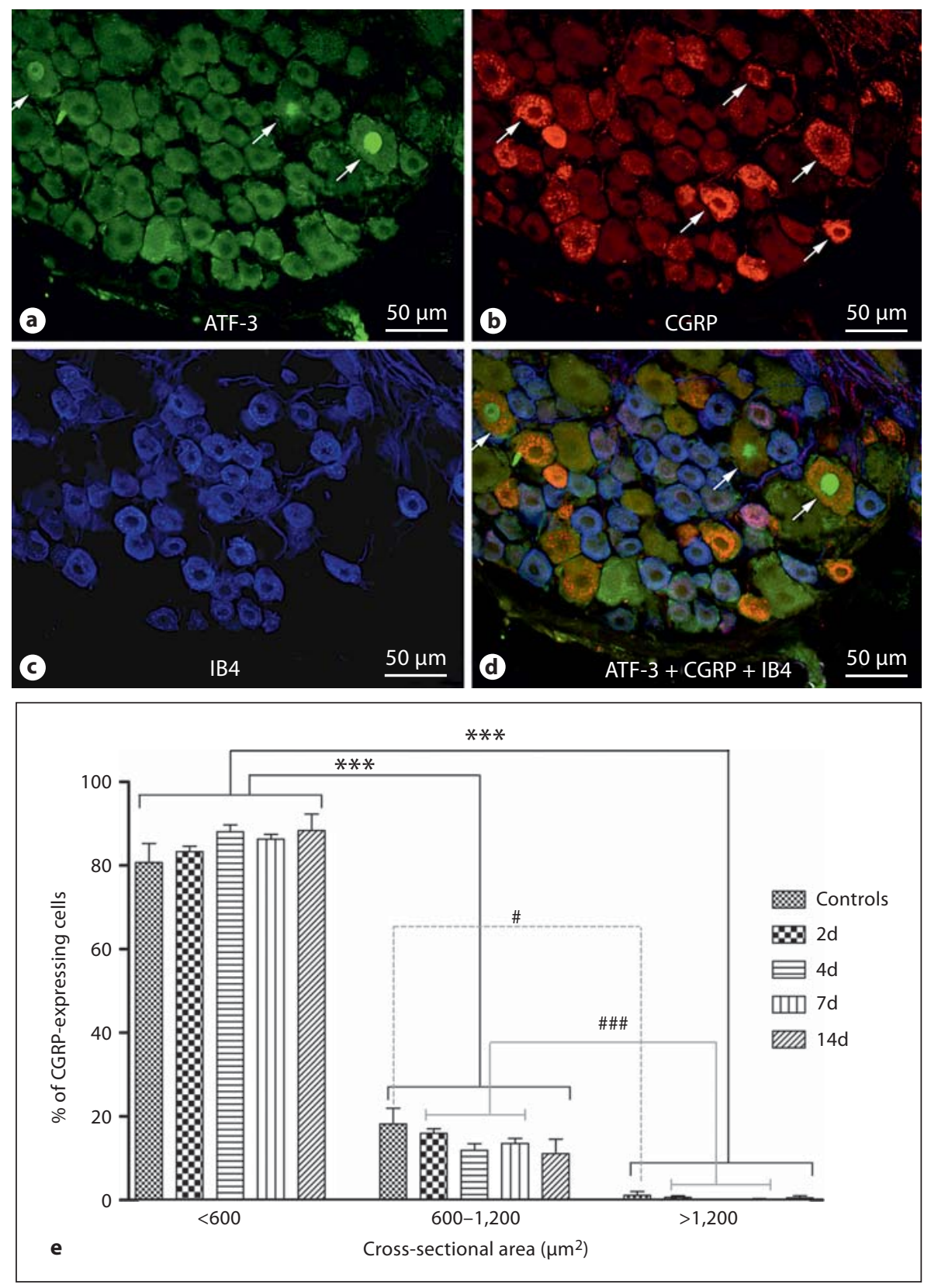

2.4\% ATF-3-IR cells) MA animals were the ones showing greater and significantly different values in comparison to vehicle-injected rats $(\mathrm{p}<0.01$ and $\mathrm{p}<0.001$ for the $2 \mathrm{~d}$ and $4 \mathrm{~d}$ MA, respectively; one-way ANOVA followed by Bonferroni post-hoc test; table 1).

Colocalization percentages for CGRP (marker of peptidergic primary afferents) and ATF-3 within the ATF-3positive neuronal population (fig. $2 \mathrm{~d}$; table 1 ) were low in MA rats, especially at later stages of disease (low at 7 days and no colocalization at 14 days; table 1). As expected, no colocalization was found for controls, where no ATF-3 expression was observed. However, statistically significant differences were detected for the population of ATF3 cells also expressing CGRP. Indeed, colocalization between ATF-3 and CGRP was significantly different for $2 \mathrm{~d}$ MA (16.0 $\pm 3.7 \%$ CGRP+ATF-3-IR cells in the ATF-3 neuronal population) and $4 \mathrm{~d}$ MA $(26.7 \pm 2.4 \%)$ when comparing either to $7 \mathrm{~d}$ or to $14 \mathrm{~d}$ MA rats (table 1). These 
Table 1. Percentage (mean \pm SEM) of ATF-3-, CGRP- and IB4-expressing cells in the total DRG neuronal population, and of ATF-3IR cells also expressing CGRP or IB4 (double labeling), in $\mathrm{L}_{5}$ DRGs from controls (vehicle-injected) and MA rats at 2, 4, 7 and 14 days post-CFA intra-articular injection

\begin{tabular}{|c|c|c|c|c|c|c|c|c|c|c|}
\hline & Controls & $\mathrm{n}$ & 2d MA & $\mathrm{n}$ & 4d MA & $\mathrm{n}$ & 7d MA & $\mathrm{n}$ & $14 \mathrm{~d}$ MA & $\mathrm{n}$ \\
\hline \% DRG cells expressing ATF-3 & 0 & 5 & $6.1 \pm 1.0^{*}$ & 4 & $8.0 \pm 2.4^{* *}$ & 5 & $2.1 \pm 0.6$ & 4 & $1.7 \pm 0.4$ & 4 \\
\hline$\%$ DRG cells expressing CGRP & $33.0 \pm 2.6$ & 5 & $32.0 \pm 4.0$ & 4 & $31.6 \pm 1.3$ & 5 & $27.4 \pm 2.4$ & 4 & $25.7 \pm 2.6$ & 4 \\
\hline$\%$ DRG cells expressing IB4 & $50.8 \pm 2.9$ & 5 & $45.1 \pm 4.1$ & 4 & $36.8 \pm 2.7$ & 5 & $47.1 \pm 4.5$ & 4 & $52.3 \pm 7.3$ & 4 \\
\hline$\%$ ATF- 3 cells expressing IB 4 & $\mathrm{~N} / \mathrm{A}$ & 5 & $3.8 \pm 1.5$ & 4 & $7.0 \pm 2.6$ & 5 & $11.5 \pm 7.9$ & 4 & $4.2 \pm 4.2$ & 4 \\
\hline
\end{tabular}

Colocalization between ATF-3 and the neuronal markers used was lower than expected; however, significant differences were found in the colocalization with CGRP for the $2 \mathrm{~d}$ and $4 \mathrm{~d}$ MA rats. Similar differences were never found for colocalization of ATF-3 with IB4. * Significant differences to control. \# Significant differences to 7d MA. ${ }^{\$}$ Significant differences to $14 \mathrm{~d}$ MA. * or ${ }^{*}$ represents p < 0.05; ${ }^{* *}$ or ${ }^{\S \S}$ represents $\mathrm{p}<0.01{ }^{\# \# \#}$ or ${ }^{\S \S}$ represents $\mathrm{p}<0.001$. One-way ANOVA followed by Bonferroni post-hoc test.

enhanced colocalizations for the $2 \mathrm{~d}$ and $4 \mathrm{~d}$ MA reflect the increases in ATF-3 expression during MA at these timepoints, as described above (fig. 1c; table 1). On the other hand, colocalization percentages of ATF-3 and IB4 (marker of non-peptidergic primary afferents) within the ATF-3-positive neuronal population were very low (much lower than ATF-3 and CGRP colocalization) and did not show any significant differences (fig. 2d; table 1).

Quantification of CGRP or IB4 (fig. 2b, c; table 1) single labeled IR cells in ipsilateral ganglia revealed no statistically significant differences in their expression during MA. Also, no noticeable changes in CGRP labeling intensity were seen.

\section{CGRP-Expressing Cells Do Not Undergo a Neuronal}

Population Switch during MA

The majority of CGRP-expressing cells, in respect to the total CGRP-positive neuronal population, were smallsized neurons (above 80\% for all experimental groups), and a smaller portion were medium-sized neurons (around 15\%; no significant differences between experimental groups). On the other hand, the percentage of larger CGRP neurons was nearly insignificant (fig. 2e). This pattern for the size distribution of CGRP-IR cells was also observed when analyzing in respect to the total DRG neuronal population (data not shown).

Possible changes in the pattern of size distribution of CGRP-expressing neurons in response to MA were also investigated, but these were not found. In fact, percentage values (both in respect to CGRP-positive or to the total DRG neuronal populations) were very similar when comparing all the different experimental groups (fig. 2e; table 1).

\section{ATF-3 and pAkt Colocalization Has Not Changed during $M A$}

The number of pAkt-IR cells was relatively high at baseline, with ipsilateral $\mathrm{L}_{5}$ DRGs from control animals showing $52.3 \pm 3.0 \%$ pAkt-IR cells in the total DRG neuronal population (fig. $3 \mathrm{~b}, \mathrm{~d}$ ). This number was maintained quite constant throughout the earlier timepoints of MA $(50.2 \pm 2.0$ and $55.0 \pm 1.3 \%$ for the 2 and 4 days of MA, respectively; fig. 3d), and showed a minor decrease in the later timepoints $(44.2 \pm 3.5$ and $48.0 \pm$ $4.3 \%$ for the 7 and 14 days of MA, respectively; fig. 3d), that did not reach statistical significance ( $p>0.05$, oneway ANOVA).

In what concerns pAkt colocalization with ATF-3 (fig. 3c, e), the quantification revealed that the percentage of double-labeled cells in respect to the total population of ATF-3-positive neurons achieved values around 55\% $(50.4 \pm 4.3,55.8 \pm 5.6,47.8 \pm 14.2$ and $64.6 \pm 4.4$ for the 2, 4,7 and 14 days of MA rats, respectively). This value has not significantly changed between MA inflamed animals, as can be easily observed in figure 3 e. No colocalization was found for controls, where none ATF-3 expression was observed.

\section{Ketoprofen Treatment Did Not Affect ATF-3}

Induction during MA but Increased CGRP Expression

Ketoprofen-treated MA rats showed significantly $(\mathrm{p}<$ $0.01)$ smaller paw diameters $(1.1 \pm 0.1$ and $1.0 \pm 0.0 \mathrm{~cm}$ for $4 \mathrm{dMA}+2 \mathrm{dKet}$ and $4 \mathrm{dMA}+4 \mathrm{dKet}$, respectively) in comparison with respective untreated MA animals (1.2 $\pm 0.0 \mathrm{~cm}$ for $4 \mathrm{~d} \mathrm{MA}$ ), although main inflammatory signs, as some swelling, redness and still some reluctance to place weight over the affected limb, remained. How- 


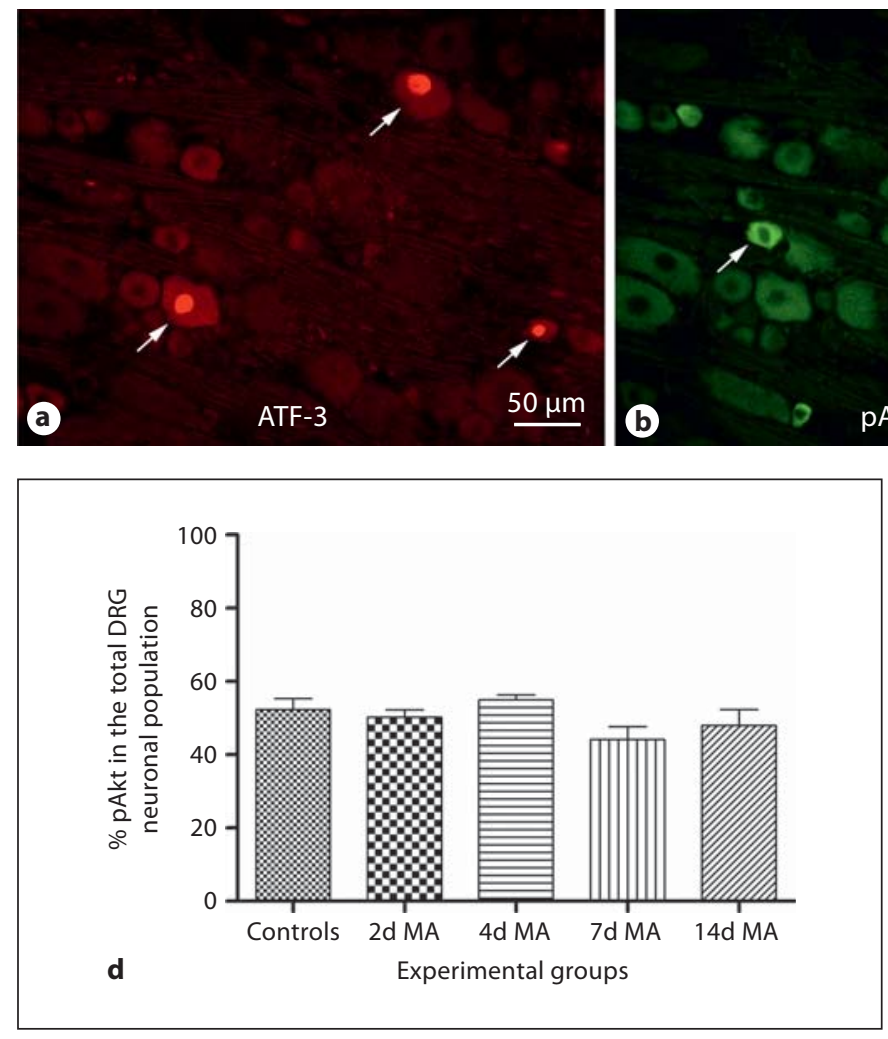

Fig. 3. pAkt expression in primary afferents of MA rats and colocalization with ATF-3. a-c Fluorescent microscope photo images depicting single immunolabeling for ATF-3 (red nuclei pointed with white arrows) (a), pAkt (green cytoplasms pointed with white arrows) (b), and double immunolabeled cells (red nuclei and green cytoplasms pointed with white arrows) (c) in a $\mathrm{L}_{5}$ ganglia from a $4 \mathrm{~d}$ MA rat. Scale bar represents $50 \mu \mathrm{m}$. d Quantification of the percentage (\%) of single labeled cells for pAkt (pAkt-IR

ever, no differences for ATF-3 expression were found (fig. 4g) in MA animals subjected either to 2 (fig. 4c) or 4 days (fig. $4 \mathrm{e})$ of ketoprofen daily injection $(6.4 \pm 1.2$ and $8.1 \pm 1.4 \%$ for $4 \mathrm{dMA}+2 \mathrm{dKet}$ and $4 \mathrm{dMA}+4 \mathrm{dKet}$, respectively) when compared to MA animals with no anti-inflammatory treatment ( $5.7 \pm 0.7 \%$; fig. $4 \mathrm{a})$. Contralateral ATF-3 expression in $\mathrm{L}_{5}$ DRGs from the ketoprofentreated MA experimental groups was null in accordance to what we had previously observed for MA animals (data not shown).

Regarding CGRP expression (fig. 4h), MA untreated animals showed significantly lower values $(31.6 \pm 1.3 \%$; fig. $4 \mathrm{~b})$ when compared to MA animals treated for both $2(43.5 \pm 2.2 \%$; fig. $4 \mathrm{~d})$ or 4 days with ketoprofen $(37.8 \pm$ 2.3\%; fig. 4f).

ATF-3 Expression during Monoarthritis
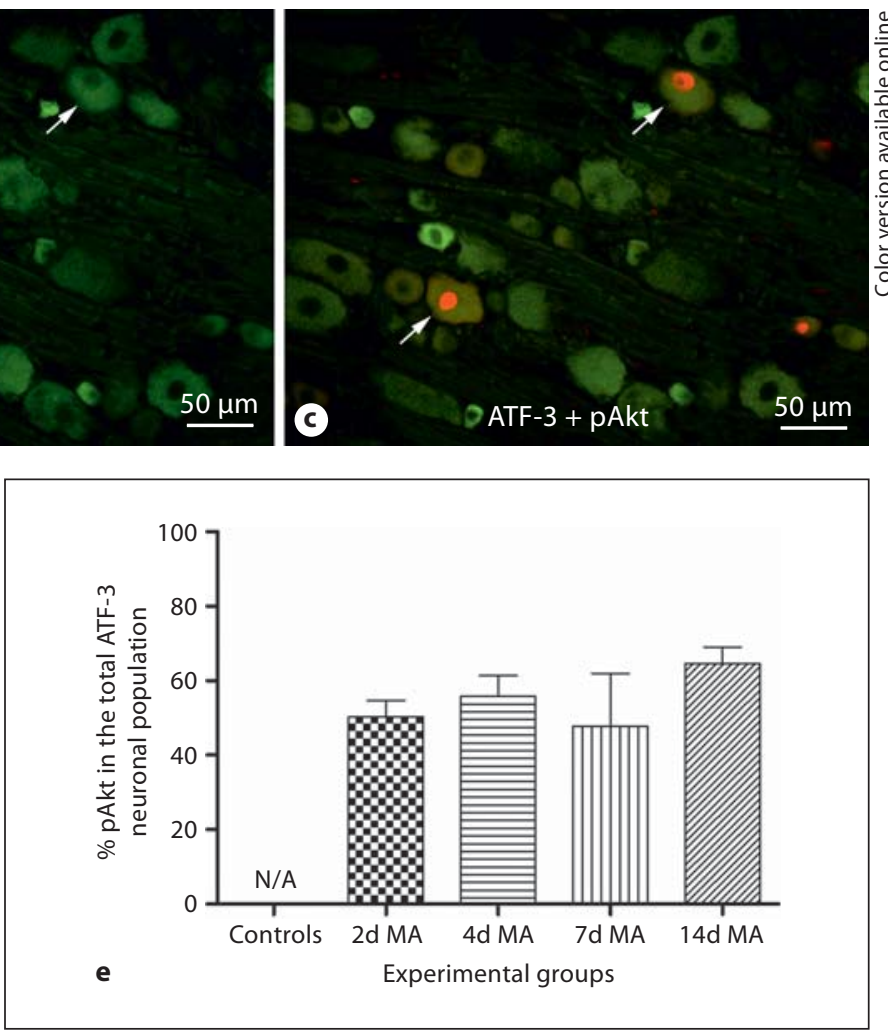

cells) in the total DRG neuronal population, in $\mathrm{L}_{5}$ ipsilateral DRGs, revealed no statistically significant differences among the experimental groups. e Percentage (\%) of colocalization between ATF-3 and pAkt did not show any significant difference during disease progression. All values shown as mean \pm SEM. One-way ANOVA followed by Bonferroni's test. $\mathrm{n}=5$ rats for each of the MA experimental groups and $n=4$ rats for the control group.

\section{Discussion}

In this study, it is reported for the first time that CFA intra-articular injection induces an immediate and transient ATF-3 expression in ipsilateral DRGs. ATF-3 expression occurs mainly in small-to-medium peptidergic neurons. No relevant colocalization was found for ATF-3 and pAkt, suggesting ATF-3 is not implicated in a survival pathway involving pAkt during MA. Finally, administration of an anti-inflammatory drug did not reverse ATF-3-induced expression in MA.

As ATF-3 is regarded as a marker of neuronal lesion [12], data suggest that some degree of neuronal damage is probably occurring, at least in an initial phase of the disease. This is supported by studies in the MIA-induced osteoarthritis model (in the knee joint) where a great ex- 
Fig. 4. Effect of ketoprofen treatment on ATF-3 and CGRP expression. a-f Fluorescent microscope photo images depicting single immunolabeling for ATF-3 (red-labeled nuclei pointed with white arrows) (a, c, e) and single immunolabeling for CGRP (red-labeled cytoplasms pointed with white arrows) (b, d, f), in $\mathrm{L}_{5}$ DRGs of $4 \mathrm{~d}$ MA rats injected with ketoprofen vehicle (a, b), 4d MA rats with 2d of ketoprofen administration $(\mathbf{c}, \mathbf{d})$ and $4 \mathrm{~d}$ MA rats with $4 \mathrm{~d}$ of ketoprofen administration $(\mathbf{e}, \mathbf{f})$. g ATF-3 expression was still induced in MA animals upon treatment with the antiinflammatory drug. No significant differences were found between any groups. Values shown as mean \pm SEM. One-way ANOVA followed by Newman-Keuls multiple comparison. $\mathrm{n}=4$ for each of the three experimental groups. $\mathbf{h}$ The number of CGRP-IR cells was significantly increased in the $\mathrm{L}_{5}$ ganglia belonging to both experimental groups with anti-inflammatory treatment. Values shown as mean \pm SEM. ${ }^{*}$ represents $\mathrm{p}<0.05$ and ${ }^{* *}$ represents $\mathrm{p}<0.01$. One-way ANOVA followed by Newman-Keuls multiple comparison. $\mathrm{n}=5$ for each of the three experimental groups.
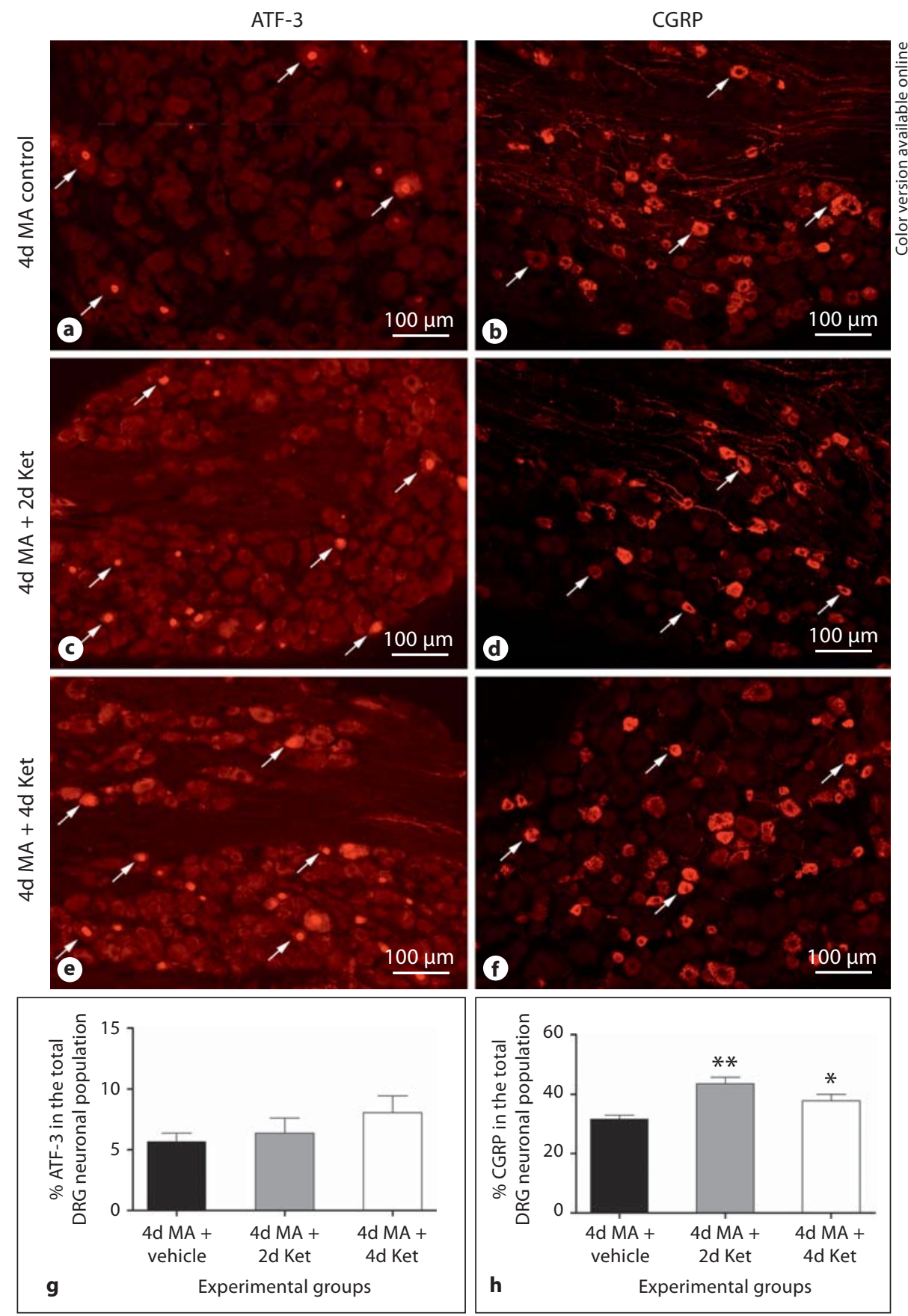

pression of ATF-3, suggestive of possible neuropathy in the early phase of the disease, was observed [14]. Controversially, others showed that antigen-induced arthritis in the knee did not induce ATF-3 expression, although there was great macrophage infiltration, implying that profound inflammation by itself cannot trigger ATF-3 induction [17]. It seems that peripheral injection of different chemical stimuli induced a stable, time- and dose-dependent significant ATF-3 expression in DRG neurons, while intraplantar injection of CFA failed to evoke ATF-3 induction [15]. Considering our data in MA, it appears that CFA only triggers ATF-3 expression in primary afferents when injected in joints. The intra-articular injection procedure per se does not seem to induce nerve damage, 
since no ATF-3 expression was detected in controls. Thus, the only possible neuronal damage inducer is likely to be CFA itself. This may also be related to specific sensitization of joint nociceptors, different for those found in skin [1]. In fact, most studies focus on cutaneous nociception, while joint pain mechanisms are not fully clarified. Chronic pain incidence in deeper tissues and joints might actually reflect enhanced vulnerability of the anatomical structures involved [1]. Additionally, as ATF-3 expression is triggered by TNF- $\alpha$ and IL- $[3,34]$, it is likely that the neuroinflammatory environment generated in response to CFA leads to local nerve damage in the joint and consequently induces ATF-3 expression in primary afferent neurons. In support, Dilley et al. [35] found ATF-3 was upregulated in DRGs following local nerve inflammation of intact sciatic nerves induced by wrapping oxidized cellulose saturated in CFA around the nerve. The transient ATF-3 expression also found in MA is consistent with other studies [36,37]. This temporal pattern is probably due to an ability of ATF-3 to act as a transcriptional autorepressor [38]. Alternatively, the transient pattern might be explained by the occurrence of regeneration mechanisms, which in fact have already been associated with ATF-3 expression [39, 40].

Cell size measurement of ATF-3-expressing neurons indicated they are mainly distributed among small-tomedium populations in $\mathrm{L}_{5}$ DRGs from MA rats. This size distribution was relatively constant throughout disease progression. Significant differences within each experimental group were found only at 14 days, indicating that in later phases of the disease ATF-3 is induced mainly in medium-sized neurons. However, triple immunoreactions against ATF-3 and the CGRP and IB4 neuronal markers did not show the expected colocalization with ATF-3 suggested by cell size analysis. The number of IB4IR cells did not significantly change along MA or when compared to controls. Moreover, the ATF-3 and IB4 colocalization did not suffer any changes between experimental groups, and, besides, was very low. Considering that a high number of ATF-3-positive neurons were small-sized, this low colocalization with IB4 might seem surprising. However, others found no IB4/ATF-3 double labeled cells following severe nerve damage [29].

The ATF-3 and CGRP colocalization was significantly increased at 2 and 4 days of MA, which reflects the significantly increased ATF-3 expression we found at these timepoints of disease. However, the same was not found for colocalization with IB4. Thus, this increased colocalization with CGRP probably means ATF-3 is mainly induced in peptidergic primary afferents at the early time- points of MA. However, the values of this colocalization were lower than expected if considering that a high number of ATF-3-positive neurons during MA were small-tomedium-sized. Indeed, ATF-3 expression has been associated with reduced CGRP mRNA expression after nerve crush, with CGRP being expressed only in uninjured neurons neighboring injured sensory neurons, which may paradoxically alter how CGRP is expressed in intact neurons [41]. This is a plausible explanation for the low colocalization found for ATF-3 and CGRP.

CGRP is normally released under painful stimulation potentiating nociceptive signaling [42], and plays important roles in the maintenance of both neuropathic and inflammatory pain states $[22,23,43,44]$. In MA, no significant differences in CGRP expression were found. Although increased CGRP levels are usually driven by inflammation, our data are actually in accordance with previous reports where CGRP expression in DRGs was not significantly altered until the later timepoints (21 days) in arthritic pain [43]. Additionally, there is ATF-3 expression in DRGs during MA which indicates neuronal injury is most likely occurring. As discussed earlier, it has been proposed that injured neurons may alter CGRP expression in intact neurons [41]. Furthermore, the nature of the peripheral nerve injury seems to have an effect on CGRP expression dynamics [22]. Others observed a considerable increase in CGRP release from DRGs during the development of inflammation and hyperalgesia, therefore explaining the significantly decreased number of CGRP-IR cells in primary afferents 2 days after CFA subcutaneous injection [44]. Cell size measurement of CGRPexpressing cells showed they are mostly small and that no neuronal population switch occurs, meaning CGRP is expressed in neurons with identical size profiles during MA, similar to other studies [44].

In the nervous tissue, ATF-3 has been found to enhance neurite outgrowth [39] and to increase the intrinsic growth state of injured neurons [40]. Overexpression of ATF-3 induces neurite elongation and inhibits apoptosis via Akt activation [8]. Therefore, we hypothesize that ATF-3 is induced during MA as a neuronal injury/stress factor, in order to drive cells into a survival/regeneration pathway. The percentage of pAkt-IR cells in the total neuronal population was relatively high even in control animals. This is in accordance with Pezet et al. [45] who reported that pAkt is present in almost every DRG neuron of the rat, although in low levels. pAkt expression reached values around $50 \%$ which is also supported by the study of Hökfelt's group [9], although this was performed in mice. Other studies in rat revealed this percentage only 
achieves values around 10\% [46], but a lot of controversy still remains. Besides possible species differences, the difficulty in detecting pAkt expression and the sensitivity of the methodological approaches might also be implicated [9]. pAkt individual expression did not show any significant difference among any experimental groups. While capsaicin- or carrageenan-induced inflammation seems to induce increases in pAkt activation $[9,45]$, one of the few studies on pAkt expression after peripheral nerve injury showed a strong reduction 7 days after the model induction [46]. Although MA is mainly an inflammatory model, some degree of peripheral neuronal damage seems to occur judging from ATF-3 expression, and additionally the time frame of the inflammation is distinct from that observed upon carrageenan or capsaicin. Thus, a possible overlap between inflammatory and neuropathic events might be balancing Akt phosphorylation levels in MA. Also pAkt and ATF-3 colocalization, around 55\%, did not change during MA progression. Thus, it is not probable that a survival pathway involving both Akt and ATF-3 is being activated during MA.

The upregulation of COX-2 and production of prostanoids are the central mechanisms for the higher hyperalgesia found in many models of peripheral inflammation [47]. In MA rats, administration of ketoprofen, a COX inhibitor anti-inflammatory drug, could not reverse ATF-3 expression. Thus, prostanoid production is unlikely to trigger ATF-3 induction, and consequent neuronal injury, in the MA model. Ketoprofen-treated MA animals showed slightly reduced paw diameters than untreated MA rats, though this was not reflected in ATF-3 expression (therefore suggesting neuronal damage is still present). Additionally, ketoprofen-treated rats showed higher CGRP expression, particularly in the $4 \mathrm{~d} M A+2 \mathrm{~d}$ Ket group. Staton et al. [48] observed decreased CGRP expression following oral administration of rofecoxib to CFA knee-injected rats between days 13 and 17. Separated quantification of small- or medium-sized cells, but particularly the differing treatments (ways of administration and timepoints) may account for the disagreement with our data in MA. Of note, our treatments started at the peak of ATF-3 induction, when apparently neuronal injury is occurring. With ketoprofen treatment, although inflammation is partially subsided, some degree of neuropathy still remains, since ATF-3 expression did not change. As discussed above, CGRP expression under these conditions might show distinct dynamics [22]. Additionally, considering Galeazza et al.'s studies [44] it is possible that the anti-inflammatory drug is blocking CGRP release from DRGs. These two conditions may lead to the greater values of CGRP immunoreactivity found in DRGs of ketoprofen-treated animals.

In conclusion, ATF-3 is expressed in DRGs in early stages of MA, suggesting neuronal damage is occurring. This is probably due to the neuroinflammatory environment induced by CFA intra-articular injection.

\section{Acknowledgements}

The work was supported by Doctoral Programme in Neurosciences, Faculty of Medicine, University of Porto. The authors wish to thank Prof. António Avelino for his expert help with the triple immunoreactions, and are grateful to Elisa Nova for skilled technical assistance during cryosectioning of DRGs.

\section{Disclosure Statement}

There are no personal or financial conflicts of interest in publishing these data.

\section{References}

$\checkmark 1$ Schaible HG, Richter F, Ebersberger A, Boettger MK, Vanegas H, Natura G, Vazquez E, Segond von Banchet G: Joint pain. Exp Brain Res 2009;196:153-162.

$>2$ Woolf CJ, Costigan M: Transcriptional and posttranslational plasticity and the generation of inflammatory pain. Proc Natl Acad Sci USA 1999;96:7723-7730.

$>3$ Lu D, Chen J, Hai T: The regulation of ATF3 gene expression by mitogen-activated protein kinases. Biochem J 2007;401:559-567.

$\checkmark 4$ Nobori K, Ito H, Tamamori-Adachi M, Adachi S, Ono Y, Kawauchi J, Kitajima S, Marumo F, Isobe M: ATF3 inhibits doxorubicin- induced apoptosis in cardiac myocytes: a novel cardioprotective role of ATF3. J Mol Cell Cardiol 2002;34:1387-1397.

5 Ohba N, Maeda M, Nakagomi S, Muraoka M, Kiyama H: Biphasic expression of activating transcription factor-3 in neurons after cerebral infarction. Brain Res Mol Brain Res 2003;115:147-156.

6 Francis JS, Dragunow M, During MJ: Over expression of ATF-3 protects rat hippocampal neurons from in vivo injection of kainic acid. Brain Res Mol Brain Res 2004;124:199-203.

7 Chen HM, Wang L, D'Mello SR: Inhibition of ATF-3 expression by B-Raf mediates the neuroprotective action of GW5074. J Neurochem 2008;105:1300-1312.

8 Nakagomi S, Suzuki Y, Namikawa K, KiryuSeo S, Kiyama H: Expression of the activating transcription factor 3 prevents c-Jun Nterminal kinase-induced neuronal death by promoting heat shock protein 27 expression and Akt activation. J Neurosci 2003;23:51875196.

> Shi TJ, Huang P, Mulder J, Ceccatelli S, Hökfelt T: Expression of p-Akt in sensory neurons and spinal cord after peripheral nerve injury. Neurosignals 2009;17:203212 . 
- 10 Obata K, Yamanaka H, Fukuoka T, Yi D, 22 Zheng LF, Wang R, Xu YZ, Yi XN, Zhang JW, Tokunaga A, Hashimoto N, Yoshikawa H, Noguchi K: Contribution of injured and uninjured dorsal root ganglion neurons to pain behavior and the changes in gene expression following chronic constriction injury of the sciatic nerve in rats. Pain 2003;101:65-77.

- 11 Peters CM, Ghilardi JR, Keyser CP, Kubota K, Lindsay TH, Luger NM, Mach DB, Schwei MJ, Sevcik MA, Mantyh PW: Tumor-induced injury of primary afferent sensory nerve fibers in bone cancer pain. Exp Neurol 2005; $193: 85-100$.

- 12 Tsujino H, Kondo E, Fukuoka T, Dai Y, Tokunaga A, Miki K, Yonenobu K, Ochi T, Noguchi K: Activating transcription factor 3 (ATF3) induction by axotomy in sensory and motoneurons: a novel neuronal marker of nerve injury. Mol Cell Neurosci 2000;15: 170-182.

$\checkmark 13$ Inglis JJ, Notley CA, Essex D, Wilson AW, Feldmann M, Anand P, Williams R: Collagen-induced arthritis as a model of hyperalgesia: functional and cellular analysis of the analgesic actions of tumor necrosis factor blockade. Arthritis Rheum 2007;56:40154023.

-14 Ivanavicius SP, Ball AD, Heapy CG, Westwood FR, Murray F, Read SJ: Structural pathology in a rodent model of osteoarthritis is associated with neuropathic pain: increased expression of ATF-3 and pharmacological characterisation. Pain 2007;128:272-282.

- 15 Braz JM, Basbaum AI: Differential ATF3 expression in dorsal root ganglion neurons reveals the profile of primary afferents engaged by diverse noxious chemical stimuli. Pain 2010;150:290-301.

16 Inglis JJ, Nissim A, Lees DM, Hunt SP, Chernajovsky Y, Kidd BL: The differential contribution of tumour necrosis factor to thermal and mechanical hyperalgesia during chronic inflammation. Arthritis Res Ther 2005; 7:R807-R816.

-17 Segond von Banchet G, Boettger MK, Fischer N, Gajda M, Brauer R, Schaible HG: Experimental arthritis causes tumor necrosis factor- $\alpha$-dependent infiltration of macrophages into rat dorsal root ganglia which correlates with pain-related behavior. Pain 2009;145:151-159.

18 Butler SH, Godefroy F, Besson JM, Weil-Fugazza J: A limited arthritic model for chronic pain studies in the rat. Pain 1992;48:73-81.

-19 Averill S, McMahon SB, Clary DO, Reichardt LF, Priestley JV: Immunocytochemical localization of trkA receptors in chemically identified subgroups of adult rat sensory neurons. Eur J Neurosci 1995;7:1484-1494.

20 Ryu PD, Gerber G, Murase K, Randic M: Actions of calcitonin gene-related peptide on rat spinal dorsal horn neurons. Brain Res 1988;441:357-361.

21 Blesch A, Tuszynski MH: GDNF gene delivery to injured adult CNS motor neurons promotes axonal growth, expression of the trophic neuropeptide CGRP, and cellular protection. J Comp Neurol 2001;436:399-410.
Zeng ZC: Calcitonin gene-related peptide dynamics in rat dorsal root ganglia and spinal cord following different sciatic nerve injuries. Brain Res 2008;1187:20-32.

23 Ruiz G, Banos JE: The effect of endoneurial nerve growth factor on calcitonin gene-related peptide expression in primary sensory neurons. Brain Res 2005;1042:44-52.

-24 Nascimento D, Pozza DH, Castro-Lopes JM, Neto FL: Altered expression of ATF-3 in primary afferent neurons during inflammatory pain. Eur J Pain 2009;13:S52.

25 Nascimento D, Pozza DH, Castro-Lopes JM, Neto FL: Induced expression of the neuronal injury marker ATF3 in primary afferents during monoarthritis: characterization of the neuronal populations involved; in Society for Neuroscience, 2010. Online. 2010. San Diego/CA: Program No. 586.3/ZZ9. 2010 Neuroscience Meeting Planner.

26 Zimmermann M: Ethical guidelines for investigations of experimental pain in conscious animals. Pain 1983;16:109-110.

27 Lourenco Neto F, Schadrack J, Platzer S, Zieglgansberger W, Tolle TR, Castro-Lopes JM: Expression of metabotropic glutamate receptors mRNA in the thalamus and brainstem of monoarthritic rats. Brain Res Mol Brain Res 2000;81:140-154.

28 Castro-Lopes JM, Tavares I, Tolle TR, Coito A, Coimbra A: Increase in GABAergic cells and GABA levels in the spinal cord in unilateral inflammation of the hindlimb in the rat. Eur J Neurosci 1992;4:296-301.

29 Averill S, Michael GJ, Shortland PJ, Leavesley RC, King VR, Bradbury EJ, McMahon SB, Priestley JV: NGF and GDNF ameliorate the increase in ATF3 expression which occurs in dorsal root ganglion cells in response to peripheral nerve injury. Eur J Neurosci 2004; 19:1437-1445.

30 Ferreira-Gomes J, Adaes S, Sarkander J, Castro-Lopes JM: Phenotypic alterations of neurons that innervate osteoarthritic joints in rats. Arthritis Rheum 2010;62:3677-3685.

- 31 Fukuoka T, Kondo E, Dai Y, Hashimoto N, Noguchi K: Brain-derived neurotrophic factor increases in the uninjured dorsal root ganglion neurons in selective spinal nerve ligation model. J Neurosci 2001;21:4891-4900.

32 Neto FL, Carvalhosa AR, Ferreira-Gomes J, Reguenga C, Castro-Lopes JM: Delta opioid receptor mRNA expression is changed in the thalamus and brainstem of monoarthritic rats. J Chem Neuroanat 2008;36:122-127.

33 Schadrack J, Neto FL, Ableitner A, CastroLopes JM, Willoch F, Bartenstein P, Zieglgansberger W, Tolle TR: Metabolic activity changes in the rat spinal cord during adjuvant monoarthritis. Neuroscience 1999;94: 595-605.
4 Inoue K, Zama T, Kamimoto T, Aoki R, Ikeda Y, Kimura H, Hagiwara M: TNF $\alpha$-induced ATF3 expression is bidirectionally regulated by the JNK and ERK pathways in vascular endothelial cells. Genes Cells 2004; 9:59-70.

35 Dilley A, Lynn B, Pang SJ: Pressure and stretch mechanosensitivity of peripheral nerve fibres following local inflammation of the nerve trunk. Pain 2005;117:462-472.

36 Chen BP, Liang G, Whelan J, Hai T: ATF3 and ATF3 delta Zip. Transcriptional repression versus activation by alternatively spliced isoforms. J Biol Chem 1994;269:15819-15826.

-37 Liang G, Wolfgang CD, Chen BP, Chen TH, Hai T: ATF3 gene. Genomic organization, promoter, and regulation. J Biol Chem 1996; 271:1695-1701.

-38 Wolfgang CD, Liang G, Okamoto Y, Allen AE, Hai T: Transcriptional autorepression of the stress-inducible gene ATF3. J Biol Chem 2000;275:16865-16870.

-39 Seijffers R, Allchorne AJ, WoolfCJ: The transcription factor ATF-3 promotes neurite outgrowth. Mol Cell Neurosci 2006;32:143-154.

-40 Seijffers R, Mills CD, Woolf CJ: ATF3 increases the intrinsic growth state of DRG neurons to enhance peripheral nerve regeneration. J Neurosci 2007;27:7911-7920.

-41 Li XQ, Verge VM, Johnston JM, Zochodne DW: CGRP peptide and regenerating sensory axons. J Neuropathol Exp Neurol 2004;63: 1092-1103.

42 Seybold VS: The role of peptides in central sensitization. Handb Exp Pharmacol 2009; 194:451-491.

43 Calza L, Pozza M, Zanni M, Manzini CU, Manzini E, Hokfelt T: Peptide plasticity in primary sensory neurons and spinal cord during adjuvant-induced arthritis in the rat: an immunocytochemical and in situ hybridization study. Neuroscience 1998;82:575-589.

44 Galeazza MT, Garry MG, Yost HJ, Strait KA, Hargreaves KM, Seybold VS: Plasticity in the synthesis and storage of substance $P$ and calcitonin gene-related peptide in primary afferent neurons during peripheral inflammation. Neuroscience 1995;66:443-458.

45 Pezet S, Spyropoulos A, Williams RJ, McMahon SB: Activity-dependent phosphorylation of Akt/PKB in adult DRG neurons. Eur J Neurosci 2005;21:1785-1797.

46 Xu JT, Tu HY, Xin WJ, Liu XG, Zhang GH, Zhai CH: Activation of phosphatidylinositol 3-kinase and protein kinase B/Akt in dorsal root ganglia and spinal cord contributes to the neuropathic pain induced by spinal nerve ligation in rats. Exp Neurol 2007;206: 269-279.

-47 Marchand F, Perretti M, McMahon SB: Role of the immune system in chronic pain. Nat Rev Neurosci 2005;6:521-532.

-48 Staton PC, Wilson AW, Bountra C, Chessell IP, Day NC: Changes in dorsal root ganglion CGRP expression in a chronic inflammatory model of the rat knee joint: differential modulation by rofecoxib and paracetamol. Eur J Pain 2007;11:283-289. 